\title{
On the First aff $(1)$-Relative Cohomology of the Lie Algebra of Vector Fields and Differential Operators
}

\section{Meher $\mathbf{A}^{*}$}

Département de Mathématiques, Faculté des Sciences de Sfax, BP 802, 3038 Sfax, Tunisie

\begin{abstract}
Let $\operatorname{Vect}\left(\mathbb{R} \mathbb{P}^{1}\right)$ be the Lie algebra of smooth vector fields on $\mathbb{R} \mathbb{P}^{1}$. In this paper, we classify aff(1) -invariant linear differential operators from $\operatorname{Vect}\left(\mathbb{R P}^{1}\right)$ to $\mathcal{D}_{\lambda, u ; v}$ vanishing on afff(1), where $\mathcal{D}_{\lambda, u ; v}:=$ Homdiff $\left(\mathcal{F}_{\lambda} \otimes \mathcal{F}_{\mu} ; \mathcal{F}_{v}\right)$ is the space of bilinear differential operators acting on weighted densities. This result allows us to compute the first differential aff(1)-relative cohomology of $\operatorname{Vect}\left(\mathbb{R P}^{1}\right)$ with coefficients in $\mathcal{D}_{\lambda, u, v}$
\end{abstract}

Keywords: Differential operators; Transvectants; Lie algebra; Cohomology

\section{Introduction}

Let $\mathfrak{g}$ be a Lie algebra and let $\mathcal{M}$ and $\mathcal{N}$ be two $\mathfrak{g}$-modules. It is wellknown that nontrivial extensions of $\mathfrak{g}$-modules:

$$
0 \rightarrow \mathcal{M} \rightarrow . \rightarrow \mathcal{N} \rightarrow 0
$$

are classified by the first cohomology group $\mathrm{H}^{1}(\mathfrak{g} ; \operatorname{Hom}(\mathcal{N}, \mathcal{M}))$ [1] Any 1-cocycle $\mathcal{L}$ generates a new action on $\mathcal{M} \oplus \mathcal{N}$ as follows: for all $g \in \mathfrak{g}$ and for all $(a, b) \in \mathcal{M} \oplus \mathcal{N}$, we define $g^{*}(a, b):=\left(g^{*} a+C^{s t} \mathcal{L}(b), g^{*} b\right)$. For the space of tensor density of weight $\lambda, \mathcal{F}_{\lambda}$, viewed as a module over the Lie algebra of smooth vector fields $\operatorname{Vect}\left(\mathbb{R P}^{1}\right)$, the classification of nontrivial extensions

$$
0 \rightarrow \mathcal{F}_{\mu} \rightarrow . \rightarrow \mathcal{F}_{\lambda} \rightarrow 0
$$

leads Feigin and Fuks [2] to compute the cohomology group $\mathrm{H}^{1}\left(\operatorname{Vect}\left(\mathbb{R} \mathbb{P}^{1}\right) ; \operatorname{Hom}\left(\mathcal{F}_{\lambda}, \mathcal{F}_{1}\right)\right)$. Later, Ovsienko and Bouarroudj [3] have computed the corresponding relative cohomology group with respect to $\mathfrak{s l}(2, \mathbb{R})$, namely

$\mathrm{H}^{1}\left(\operatorname{Vect}\left(\mathbb{R} \mathbb{P}^{1}\right), \mathfrak{s l}(2, \mathbb{R}) ; \operatorname{Hom}\left(\mathcal{F}_{\lambda}, \mathcal{F}_{\mu}\right)\right)$.

In this paper, we will compute the first cohomology group

$\mathrm{H}^{1}\left(\operatorname{Vect}\left(\mathbb{R P}^{1}\right), \mathfrak{a f f}(1) ; \operatorname{Hom}\left(\mathcal{F}_{\lambda} \otimes, \mathcal{F}_{\mu}, \mathcal{F}_{v}\right)\right)$.

\section{$\operatorname{Vect}(\mathbb{R})$-Module Structures on the Space of Bilinear Differential Operators}

Consider the standard (local) action of $\mathfrak{a f f}(1)$ on $\mathbb{R}$ by linearfractional transformations. Although the action is local, it generates global vector fields

$$
\left\{\frac{d}{d x}, x \frac{d}{d x}\right\},
$$

that form a Lie subalgebra of $\operatorname{Vect}(\mathbb{R})$ isomorphic to the Lie algebra $\mathfrak{a} \mathfrak{f} \mathfrak{f}(1)$. This realization of $\mathfrak{a} \mathfrak{f} \mathfrak{f}(1)$ is understood throughout this paper.

\section{The space of tensor densities on $\mathbb{R} \mathbb{P}^{1}$}

The space of tensor densities of weight $\lambda$ (or $\lambda$-densities) on $\mathbb{R P}^{1}$, denoted by:

$$
\mathcal{F}_{\lambda}=\left\{f(d x)^{\lambda} \mid f \in C^{\infty}(\mathbb{R})\right\}, \lambda \in \mathbb{R},
$$

is the space of sections of the line bundle $\left(T^{*} \mathbb{R} \mathbb{P}^{1}\right)^{\otimes^{\lambda}}$. This space coincides with the space of functions and differential forms for $\lambda=0$ and for $\lambda=1$, respectively. The Lie algebra $\operatorname{Vect}\left(\mathbb{R P}^{1}\right)$ acts on $\mathcal{F}_{\lambda}$ by the Lie derivative. For all $X \in \operatorname{Vect}\left(\mathbb{R P P}^{1}\right)$ and for all $\varphi \in \mathcal{F}_{\lambda}$ :

$$
L_{X}\left(\varphi(d x)^{\lambda}\right)=X \varphi^{\prime}+\lambda \varphi X^{\prime},
$$

where the superscript ' stands for $d / d x$.

The space of bilinear differential operators as a $\operatorname{Vect}\left(\mathbb{R P}^{1}\right)$ module

We are interested in defining a three-parameter family of $\operatorname{Vect}\left(\mathbb{R P}^{1}\right)$-modules on the space of bilinear differential operators. The counterpart $\operatorname{Vect}\left(\mathbb{R P}^{1}\right)$-modules of the space of linear differential operators is a classical object [4].

Consider bilinear differential operators that act on tensor densities:

$$
A: \mathcal{F}_{\lambda} \otimes \mathcal{F}_{\mu} \rightarrow \mathcal{F}_{v}
$$

The Generalized Lie algebra $\operatorname{Vect}\left(\mathbb{R P}^{1}\right)$ acts on the space of bilinear differential operators as follows. For all $\varphi \in \mathcal{F}_{\lambda}$ and for all $\psi \in \mathcal{F}_{\mu}$ :

$$
L_{X}^{\lambda, \mu ; \nu}(A)(\phi, \psi)=L_{X}^{v} \circ A(\phi, \psi)-A\left(L_{X}^{\lambda} \phi, \psi\right)-A\left(\phi, L_{X}^{\mu} \psi\right)
$$

where $L_{X}^{\lambda}$ is the action (1). We denote by $\mathcal{D}_{\lambda, \mu ; \nu}$ the space of bilinear differential operators (2) endowed with the defined $\operatorname{Vect}\left(\mathbb{R} \mathbb{P}^{1}\right)$-module structure (3).

\section{Relative Cohomology}

Let us first recall some fundamental concepts from cohomology theory [1]. Let $g$ be a Lie algebra acting on a vector space $V$ and let $h$ be a sub- algebra of $g$. (If $h$ is omitted it assumed to be $\{0\}$.) The space of $h$-relative $n$-cochains of $g$ with values in $V$ is the $g$-module

$C^{n}(g, h ; V):=\operatorname{Hom}_{h}\left(\Lambda^{n}(g / h) ; V\right)$

The coboundary operator $\delta_{n}: C^{n}(g, h ; V) \rightarrow C^{n+1}(g, h ; V)$ is a $g$-map satisfying ${ }_{n}^{\circ} \delta_{n 1}=0$. The kernel of $\delta_{n}$, denoted $Z^{n}(\mathfrak{g}, h ; V)$, is the space of $h$-relative $n$-cocycles, among them, the elements in the range of $\delta$ are called $h$-relative $n$ - coboundaries. We denote $B^{n}(\mathfrak{g}, h ; V)$ the space of $n$-coboundaries.

*Corresponding author: Meher A, Département de Mathématiques, Faculté des Sciences de Sfax, BP 802, 3038 Sfax, Tunisie, Tel: 71872 600; Fax: 71871 666; E-mail: abdaoui.meher@gmail.com

Received April 08, 2017; Accepted June 21, 2017; Published June 27, 2017

Citation: Meher A (2017) On the First aff(1)-Relative Cohomology of the Lie Algebra of Vector Fields and Differential Operators. J Generalized Lie Theory Appl 11: 269. doi: $10.4172 / 1736-4337.1000269$

Copyright: (c) 2017 Meher A. This is an open-access article distributed under the terms of the Creative Commons Attribution License, which permits unrestricted use, distribution, and reproduction in any medium, provided the original author and source are credited. 

space

By definition, the $n^{\text {th }} h$-relative cohomolgy space is the quotient

$$
\mathrm{H}^{n}(\mathfrak{g}, h ; V)=Z^{n}(\mathfrak{g}, h ; V) / B^{n}(\mathfrak{g}, h ; V) .
$$

We will only need the formula of $\delta_{n}$ (which will be simply denoted $\delta$ ) in degrees 0,1 and 2 : for $v \in C^{0}(g, h ; V)^{n}=V^{h}, \delta v(g):=(-1)^{|g||v|} g . v$, where

$V^{h}=\{v \in V \mid h . v=0$ for all $h \in h\}$,

and for $\Upsilon \in C^{1}(g, h ; V)$,

$\delta(\Upsilon)(x, y):=x \cdot \Upsilon(y)-y \cdot \Upsilon(x)-\Upsilon([x, y])$ for any $x, y \in g$.

\section{$\mathfrak{a} \mathfrak{f} \mathfrak{f}(1)$-Invariant Differential Operators}

The following steps to compute the relative cohomology has intensively been used in refs. [3,5-8]. First, we classify afff(1)-invariant differential operators, then we isolate among them those that are 1 -cocycles. To do that, we need the following Lemma.

\section{Lemma 4.1}

Any 1-cocycle vanishing on the subalgebra aff $(1)$ of $\operatorname{Vect}(\mathbb{R})$ is $\mathfrak{a f f}(1)$-invariant.

The 1-cocycle condition of $\Upsilon$ reads:

$$
X \cdot \Upsilon(Y)-Y \cdot \Upsilon(X)-\Upsilon([X, Y])=0,
$$

where $X, Y \in \operatorname{Vect}\left(\mathbb{R P}^{1}\right)$. Thus, if $\Upsilon(X)=0$ for all $X \in$ aff(1), eqn. (4) becomes

$$
\Upsilon([X, Y])=X \cdot \Upsilon(Y)
$$

expressing the $\mathfrak{a} \mathfrak{f} f(1)$-invariance property of $\Upsilon$.

As our 1-cocycles vanish on aff(1), we will investigate aff(1)invariant linear differential operators that vanish on aff(1).

Proposition 4.2: There exist afff(1)-invariant bilinear differential operators $J_{k}^{\lambda, \mu}: \mathcal{F}_{\lambda} \otimes \mathcal{F}_{\mu} \rightarrow \mathcal{F}_{\lambda+\mu+k}$ given by:

$$
J_{k}^{\lambda, \mu}\left(\varphi d x^{\lambda}, \phi d x^{\mu}\right)=\sum_{i+j=k} \gamma_{i, j} \varphi^{(i)} \phi^{(j)} d x^{\lambda+\mu+k}
$$

where $k \in \mathbb{N}$ and the coefficients $\gamma_{i, j}$ are constants.

Proof. Any differential operator $J_{k}^{\lambda, \mu}: \mathcal{F}_{\lambda} \otimes \mathcal{F}_{\mu} \rightarrow \mathcal{F}_{v}$ is of the form

$$
J_{k}^{\lambda, \mu}\left(f d x^{\lambda}, g d x^{\mu}\right)=\sum_{n=0 i+j=k}^{m} \sum_{i, j} f^{(i)} g^{(j)} d x^{\nu}, \quad m \in \mathbb{N}
$$

The $\operatorname{ssp}(1 \mid 2)$-invariant property of the operators $J_{k}^{\lambda, \mu}$ with respect to the vector field $X=x \frac{d}{d x}$ yields:

$$
\frac{d}{d x} \gamma_{i, j}=0 \quad \text { and } \quad v-\lambda-\mu=k \quad \text { with } \quad k=i+j .
$$

So, we see that the corresponding operator can be expressed as (5).

Proposition 4.3: There exist afff(1)-invariant trilinear differential operators $K_{k}^{\tau, \lambda, \mu}: \mathcal{F}_{\tau} \otimes \mathcal{F}_{\lambda} \otimes \mathcal{F}_{\mu} \rightarrow \mathcal{F}_{\tau+\lambda+\mu+k}$ given by:

$$
K_{k}^{\tau, \lambda, \mu}(\varphi, \phi, \psi)=\sum_{i+j+l=k} \gamma_{i, j, l} \varphi^{(i)} \phi^{(j)} \psi^{(l)} .
$$

where $i+j+l=k$ and the coefficients $\gamma_{i, j, l}$ are constants.

If $\tau, \lambda$ and $\mu$ are generic, then the space of solutions is $\frac{1}{2}(k+1)(k+2)$ -dimensional.

Proposition 4.4: There exist $\mathfrak{a f f}(1)$-invariant trilinear differential operators $K_{k}^{\lambda, \mu}: \operatorname{Vect}\left(\mathbb{R P}^{1}\right) \otimes \mathcal{F}_{\lambda} \otimes \mathcal{F}_{\mu} \rightarrow \mathcal{F}_{\lambda+\mu+k-1}$ that vanishe on $\mathfrak{a f f}(1)$ given by:

$$
K_{k}^{\lambda, \mu}(X, \phi, \psi)=\sum_{i+j+l=k} \gamma_{i, j, l} X^{(i)} \phi^{(j)} \psi^{(l)} .
$$

where $i+j+l=k$ and the coefficients $\gamma_{i, j, l}$ are constants but $\gamma_{0, j, k-j}=\gamma_{1, j, k-}$ $j-1=0$. Moreover, the space of solutions is $\frac{1}{2} k(k-1)$-dimensional, for
all $\lambda$ and $\mu$.

Proof of Proposition 4.3 and 4.4: We are going to prove Proposition 4.3 and 4.4 simultaneously. Any differential operator $K_{k}^{\tau, \lambda, \mu}: \mathcal{F}_{\tau} \otimes \mathcal{F}_{\lambda} \otimes \mathcal{F}_{\mu} \rightarrow \mathcal{F}_{\tau+\lambda+\mu+k}$ is of the form

$$
K_{k}^{\tau, \lambda, \mu}(\varphi, \phi, \psi)=\sum_{i+j+l=k} \gamma_{i, j, l} \varphi^{(i)} \phi^{(j)} \psi^{(l)} .
$$

where $\gamma_{i, j, l}$ are functions. The $\mathfrak{a f f}(1)$-invariant property of the operators $K_{k}^{\tau, \lambda, \mu}$ reads as follows.

$$
L_{X}^{v} K_{k}^{\tau, \lambda, \mu}(\phi, \varphi, \psi,)=K_{k}^{\tau, \lambda, \mu}\left(L_{X}^{\tau} \phi, \varphi, \psi\right)+K_{k}^{\tau, \lambda, \mu}\left(\phi, L_{X}^{\lambda} \varphi, \psi\right)+K_{k}^{\tau, \lambda, \mu}\left(\phi, \varphi, L_{X}^{\mu} \psi\right)
$$

The invariant property with respect to the vector field $X=\frac{d}{d}$ implies that $\gamma_{i, j, l}^{\prime}=0$. On the other hand, the invariant propetty with respect to the vector fields $X=x \frac{d}{d x}$ implies that $v=\tau+\lambda+\mu+k$. If $\tau$, $\lambda$ and $\mu$ are generic, then the space of solutions is $\frac{1}{2}(k+1)(k+2)$ -dimensional, spanned by

$$
\begin{aligned}
& \gamma_{0,0, k}, \gamma_{0,1, k-1}, \cdots, \gamma_{0, k, 0}, \\
& \gamma_{1,0, k-1}, \gamma_{1,1, k-2}, \cdots, \gamma_{1, k-1,0}, \\
& \quad \vdots \\
& \gamma_{k-1,0,1}, \gamma_{k-1,1,0}, \\
& \gamma_{k, 0,0} .
\end{aligned}
$$

Now, the proof of Proposition 4.4 follows as above by putting $\tau-1$. In this case, the space of solutions is $\frac{1}{2} k(k-1)$-dimensional, spanned by

$$
\begin{aligned}
& \gamma_{2,0, k-2}, \gamma_{2,1, k-3}, \cdots, \gamma_{2, k-2,0}, \\
& \gamma_{3,0, k-3}, \gamma_{3,1, k-4}, \cdots, \gamma_{3, k-3,0}, \\
& \vdots \\
& \gamma_{k-1,0,1}, \gamma_{k-1,1,0}, \\
& \gamma_{k, 0,0} .
\end{aligned}
$$

\section{Cohomology of $\operatorname{Vect}\left(\mathbb{R P}^{1}\right)$ acting on $\mathcal{D}_{\lambda, \mu ; \nu}$}

In this section, we will compute the first cohomology group of $\operatorname{Vect}\left(\mathbb{R P}^{1}\right)$ with values in $\mathcal{D}_{\lambda, \mu ; j}$, vanishing on $\mathfrak{a f f}(1)$. Our main result is the following:

\section{Theorem 5.1}

(i) For $v-\mu-\lambda \leq 11$, the space $H_{\text {diff }}^{1}\left(\operatorname{Vect}\left(\mathbb{R P}^{1}\right), \mathfrak{a f f}(1) ; \mathcal{D}_{\lambda, \mu ; \nu}\right)$ has the following structure:

(1) If $v-\mu-\lambda=1$, then

$$
\mathrm{H}_{\text {diff }}^{1}\left(\operatorname{Vect}\left(\mathbb{R} \mathbb{P}^{1}\right), \mathfrak{a f f}(1) ; \mathcal{D}_{\lambda, \mu ; \nu}\right) \simeq\left\{\begin{array}{l}
\mathbb{R} \text { if } 0.2 \mathrm{~cm}(\lambda, \mu)=(0,0), \\
0 \quad \text { otherwise. }
\end{array}\right.
$$

(2) If $v-\mu-\lambda=2$, then

$\mathrm{H}_{\text {diff }}^{1}\left(\operatorname{Vect}\left(\mathbb{R} \mathbb{P}^{1}\right)\right.$, aff $\left.(1) ; \mathcal{D}_{\lambda, \mu, \nu}\right) \simeq\left\{\begin{array}{ccc}0.2 \mathrm{~cm} & \mathbb{R} & \text { if }(\lambda, \mu) \in\left\{(0,0),\left(0,-\frac{1}{2}\right),\left(-\frac{1}{2}, 0\right)\right\}, 0.2 \mathrm{~cm} \\ & 0 & \text { otherwise. }\end{array}\right.$

(3) If $v-\mu-\lambda=3$, then 
$\mathrm{H}_{\text {diff }}^{\mathrm{l}}\left(\operatorname{Vect}\left(\mathbb{R} \mathbb{P}^{\prime}\right)\right.$, aff $\left.(1) ; \mathcal{D}_{\lambda, \mu, j}\right) \simeq\left\{\begin{array}{cc}0.2 \mathrm{~cm} & \mathbb{R} \quad \text { if }(\lambda, \mu) \in\left\{\begin{array}{c}(0,-1),(-1,0),\left(0,-\frac{1}{3}\right), \\ \left(-\frac{1}{3}, 0\right),\left(-\frac{1}{2},-\frac{1}{2}\right)\end{array}\right. \\ 0 & \text { otherwise. }\end{array}\right\}, 0.2 \mathrm{~cm}$

(4) If $v-\mu-\lambda=4$, then

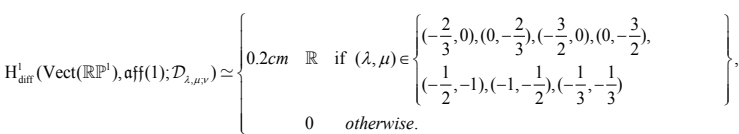

(5) If $v-\mu-\lambda=5$, then

$\mathbf{H}_{\text {diff }}^{1}\left(\operatorname{Vect}\left(\mathbb{R} \mathbb{P}^{1}\right), \mathfrak{a f f}(1) ; \mathcal{D}_{\lambda, \mu, v}\right) \simeq\left\{\begin{array}{c}(-2,0),(0,-2),(-1,0),(0,-1), \\ 0.2 c m \quad \mathbb{R} \text { if }(\lambda, \mu) \in\left\{\begin{array}{c}(-1,-1),\left(-\frac{2}{3},-\frac{1}{3}\right),\left(-\frac{1}{3},-\frac{2}{3}\right), \\ \left(-\frac{3}{2},-\frac{1}{2}\right)\end{array}\right. \\ 0 \text { otherwise. }\end{array}\right.$

(6) If $\nu-\mu-\lambda=6$, then

$\mathbf{H}_{\text {diff }}^{1}\left(\operatorname{Vect}\left(\mathbb{R} \mathbb{P}^{1}\right), \mathfrak{a f f}(1) ; \mathcal{D}_{\lambda, \mu, j}\right) \simeq\left\{\begin{array}{c}\left(-\frac{1}{2},-\frac{1}{4}\right),\left(0,-\frac{3}{4}\right),\left(-\frac{2}{3},-\frac{2}{3}\right), \\ \left(-2 c m \quad \mathbb{R} \quad \text { if }(\lambda, \mu) \in\left\{\begin{array}{c}\left.\frac{1}{3},-1\right),\left(0,-\frac{4}{3}\right),\left(-\frac{5}{2}, 0\right), \\ \left(-2,-\frac{1}{2}\right),\left(-\frac{3}{2},-1\right),\left(-\frac{1}{2},-2\right)\end{array}\right.\right. \\ 0 \quad \text { otherwise. }\end{array}\right.$

(7) If $v-\mu-\lambda=7$, then

$\mathrm{H}_{\text {diff }}^{\mathrm{l}}\left(\operatorname{Vect}\left(\mathbb{R} \mathbb{P}^{\mathrm{l}}\right)\right.$, aff $\left.(1) ; \mathcal{D}_{\lambda, \mu ; y}\right) \simeq\left\{\begin{array}{c}(0,0),\left(-\frac{1}{7}, 0\right),\left(0,-\frac{1}{7}\right), \\ 0.2 \mathrm{~cm} \quad \mathbb{R} \quad \text { if }(\lambda, \mu) \in\left\{\begin{array}{l}\left(-\frac{3}{5}, 0\right),\left(0,-\frac{3}{5}\right),(0,-1), \\ (-2,-1),\left(-\frac{1}{2},-\frac{5}{2}\right),(0,-3)\end{array}\right. \\ 0 \quad \text { otherwise. }\end{array}\right.$

(8) If $v-\mu-\lambda=8$, then

$\mathrm{H}_{\mathrm{diff}}^{1}\left(\operatorname{Vect}\left(\mathbb{R} \mathbb{P}^{\mathrm{l}}\right), \mathfrak{a f f}(1) ; \mathcal{D}_{\lambda, \mu ; v}\right) \simeq\left\{\begin{array}{c}0.2 \mathrm{~cm} \quad \mathbb{R} \quad \text { if }(\lambda, \mu) \in\left\{\begin{array}{l}(0,0),\left(0,-\frac{1}{8}\right),\left(-\frac{2}{7}, 0\right), \\ \left(0,-\frac{2}{7}\right),\left(-\frac{1}{2}, 0\right),\left(0,-\frac{1}{2}\right), \\ \left(-\frac{3}{5},-\frac{1}{5}\right),(0,-2),\left(0,-\frac{7}{2}\right)\end{array}\right. \\ 0 \quad \text { otherwise. }\end{array}\right.$

(9) If $\nu-\mu-\lambda=9$, then

$\mathrm{H}_{\text {diff }}^{1}\left(\operatorname{Vect}\left(\mathbb{R} \mathbb{P}^{1}\right)\right.$, aff $\left.(1) ; \mathcal{D}_{\lambda, \mu ; y}\right) \simeq\left\{\begin{array}{c}(0,0),\left(0,-\frac{1}{9}\right),\left(-\frac{1}{4}, 0\right), \\ 0.2 c m \quad \mathbb{R} \quad \text { if }(\lambda, \mu) \in\left\{\begin{array}{c}\left(-\frac{2}{3}, 0\right),\left(0,-\frac{2}{3}\right),\left(-\frac{3}{5},-\frac{2}{5}\right), \\ \left(0,-\frac{7}{3}\right),(0,-4)\end{array}\right. \\ 0 \quad \text { otherwise. }\end{array}\right.$

(10) If $v-\mu-\lambda=10$, then

$\mathrm{H}_{\text {diff }}^{1}\left(\operatorname{Vect}\left(\mathbb{R}^{1}\right), \mathfrak{a f f}(1) ; \mathcal{D}_{\lambda, \mu ; y}\right) \simeq\left\{\begin{array}{c}(0,0),\left(0,-\frac{1}{10}\right),\left(-\frac{2}{9}, 0\right), \\ 0.2 c m \quad \mathbb{R} \quad \text { if }(\lambda, \mu) \in\left\{\begin{array}{l}\left(-\frac{5}{6}, 0\right),\left(0,-\frac{5}{6}\right),\left(-\frac{3}{5},-\frac{3}{5}\right), \\ \left(0,-\frac{8}{3}\right),\left(0,-\frac{9}{2}\right)\end{array}\right. \\ 0 \quad \text { otherwise. }\end{array}\right.$

(11) If $v-\mu-\lambda=11$, then

$\mathrm{H}_{\mathrm{diff}}^{1}\left(\operatorname{Vect}\left(\mathbb{R} \mathbb{P}^{1}\right), \mathfrak{a f f}(1) ; \mathcal{D}_{\lambda, \mu, v}\right) \simeq\left\{\begin{array}{c}(0,0),\left(0,-\frac{1}{11}\right),\left(0,-\frac{1}{5}\right), \\ 0.2 \mathrm{~cm} \quad \mathbb{R} \quad \text { if }(\lambda, \mu) \in\left\{\begin{array}{l}\left(0,-\frac{1}{3}\right),\left(0,-\frac{5}{7}\right),\left(-\frac{7}{5}, 0\right), \\ \left(0,-\frac{7}{5}\right),\left(-\frac{1}{2},-\frac{9}{2}\right)\end{array}\right. \\ 0 \quad \text { otherwise. }\end{array}\right.$

(ii) If $\nu-\mu-\lambda$ is semi-integer but $\lambda$ and $\mu$ are generic then,

$\mathrm{H}_{\text {diff }}^{1}\left(\operatorname{Vect}\left(\mathbb{R P}^{1}\right), \mathfrak{a f f}(1) ; \mathcal{D}_{\lambda, \mu ; \nu}\right)=0$.
Proof of Theorem 5.1: To proof Theorem (5.1) we proceed bye following the three steps:

- We will investigate the dimension of the space of operators that satisfy the 1-cocycle condition. By Proposition (4.4), its dimension is at most $\frac{1}{2} k(k-1)$, where $k=v-\mu-\lambda+1$, since any 1 -cocycle that vanishes on $\mathfrak{a f f}(1)$ is certainly $\mathfrak{a f f}(1)$-invariant.

- We will study all trivial 1-cocycles, namely, operators of the form

$L_{X} B$,

where $B$ is a bilinear operator. As our 1-cocycles vanish on the Lie algebra $\mathfrak{a f f}(1)$, it follows that the operator $B$ coincides with the transvectant $J_{k}^{\lambda, \mu}$.

- By taking into account Part 1 and Part 2 and depending on $\lambda$ and $\mu$ the dimension of the cohomology group $\mathrm{H}_{\text {diff }}^{1}\left(\operatorname{Vect}\left(\mathbb{R P}^{1}\right), \mathfrak{a f f}(1) ; \mathcal{D}_{\lambda, \mu ; \nu}\right)$ will be equal to

$\operatorname{dim}($ operators that are $1-$ cocycles $)-\operatorname{dim}\left(\right.$ operators of the form $\left.L_{X} J_{k}^{\lambda, \mu}\right)$.

Now, clearly the coboundary $L_{X} J_{k}^{\lambda, \mu}$ has the following form:

$$
L_{X} J_{k}^{\lambda, \mu}(X, \phi, \psi)=\sum_{i+j+l=k+1} \beta_{i, j, l} X^{(i)} \phi^{(j)} \psi^{(l)},
$$

where

$$
\beta_{0, j, l}=\beta_{1, j, l}=0 \text {. }
$$

The following Lemma is proved directly which will be useful in the proof of Theorem 5.1.

\section{Lemma 5.2}

For $\lambda, \mu \in \mathbb{R}$

$\beta_{\alpha, \beta, k-\alpha-\beta+1}=-\left(\left(\begin{array}{c}\alpha+\beta-1 \\ \alpha\end{array}\right)+\lambda\left(\begin{array}{c}\alpha+\beta-1 \\ \alpha-1\end{array}\right)\right) \gamma_{\alpha+\beta-1, k-\alpha-\beta+1}-\left(\left(_{\alpha}^{k-\beta}\right)+\mu\left(\begin{array}{c}k-\beta \\ \alpha-1\end{array}\right)\right) \gamma_{\beta, k-\beta}$, where $\alpha \geq 2$ and $\beta \geq 0$.

We need also the following Lemma.

\section{Lemma 5.3}

Every 1-cocycle on $\operatorname{Vect}\left(\mathbb{R P}^{1}\right)$ with values in $\left.\mathcal{D}_{\lambda, \mu ; v}\right)$ is differentiable

Proof [7].

Now we are in position to prove Theorem (5.1). By Lemma (5.3), any 1 -cocycle on $\operatorname{Vect}\left(\mathbb{R P}^{1}\right)$ should retains the following general form:

$$
C(X, \phi, \psi)=\sum_{i+j+l=k} c_{i, j, l} X^{(i)} \phi^{(j)} \psi^{(l)},
$$

where $c_{i, j, l}$ are constants. The fact that this 1 -cocycle vanishes on $\mathfrak{a f f}(1)$ implies that

$$
c_{0, j, l}=c_{1, j, l}=0 \text {. }
$$

The 1-cocycle condition reads as follows: for all $\varphi \in \mathcal{F}_{\lambda}$, for all $\psi \in \mathcal{F}_{\mu}$ and for all $X \in \operatorname{Vect}\left(\mathbb{R P}^{1}\right)$, one has

$$
c([X, Y], \phi, \psi)-L_{X}^{\lambda, \mu ; v} B(Y, \phi, \psi)+L_{Y}^{\lambda, \mu ; v} B(X, \phi, \psi)=0 .
$$

The case where $\boldsymbol{v}-\boldsymbol{\mu}-\boldsymbol{\lambda}=\mathbf{1}$ : In this case, according to Proposition 4.4 , the 1-cocycle (24) can be expressed as follows:

$$
\Upsilon(X, \phi, \psi)=c_{2,0,0} X^{\prime \prime} \phi \psi \text {. }
$$

By a direct computation, we can see that the 1-cocycle condition is always satisfied. Let us study the triviality of this 1-cocycle. A direct computation proves that 


$$
L_{X} J_{1}^{\lambda, \mu}=\beta_{2,0,0} X^{\prime \prime} \phi \psi=-\left(\lambda \gamma_{1,0}+\mu \gamma_{0,1}\right) X^{\prime \prime} \phi \psi \text {. }
$$

So, for $(\lambda, \mu)=(0,0)$, the coeffcient $c_{2,0,0}$ cannot be eliminated by adding a coboundary. Hence, the cohomology space is one-dimensional. While for $(\lambda, \mu) \neq(0,0)$, we can see that the coeffcient $c_{2,0,0}$ can be eliminated because $\beta_{2,0,0} \neq 0$. Hence, the cohomology is zero-dimensional.

The case where $\boldsymbol{v}-\mu-\lambda=2$ : In this case, according to Proposition 4.4 , the 1-cocycle (24) can be expressed as follows:

$$
\Upsilon(X, \phi, \psi)=c_{3,0,0} X^{\prime \prime \prime} \phi \psi+c_{2,1,0} X^{\prime \prime} \phi^{\prime} \psi+c_{2,0,1} X^{\prime \prime} \phi \psi^{\prime} .
$$

By a direct computation, we can see that the 1-cocycle condition is always satisfied. Let us study the triviality of this 1-cocycle. A direct computation proves that

$$
L_{X} J_{2}^{\lambda, \mu}=\beta_{3,0,0} X^{\prime \prime \prime} \phi \psi+\beta_{2,1,0} X^{\prime \prime} \phi^{\prime} \psi+\beta_{2,0,1} X^{\prime \prime} \phi \psi^{\prime} .
$$

where

$$
\beta_{3,0,0}=-\lambda \gamma_{2,0}-\mu \gamma_{0,2} ; \beta_{2,1,0}=-(2 \lambda+1) \gamma_{2,0}-\mu \gamma_{1,1} \text { and } \beta_{2,0,1}=-\lambda \gamma_{1,1}-(2 \mu+1) \gamma_{0,2} \text {. }
$$

So, for $(\lambda, \mu)=(0,0),\left(-\frac{1}{2}, 0\right),\left(0,-\frac{1}{2}\right)$, the cohomology space is one-dimensional, since only one of the coefficients $c_{3,0,0}, c_{2,1,0}$ or $c_{2,0,1}$ cannot be eliminated by adding a coboundary. While for $(\lambda, \mu) \neq(0,0),\left(-\frac{1}{2}, 0\right),\left(0,-\frac{1}{2}\right)$, the coeffcient $c_{3,0,0}, \quad c_{2,1,0}$ and $c_{2,0,1}$ can be eliminated because $\beta_{3,0,0}, \beta_{2,1,0}$ and $\beta_{2,0,1}$ are nonzero. Hence, the cohomology space is zero-dimensional.

The case where $v-\mu-\lambda \geq 3$ : In this case, the 1-cocycle condition is equivalent to the system:

$$
\begin{aligned}
& \left(\left(\begin{array}{c}
\alpha+\beta-1 \\
\alpha
\end{array}\right)-\left(\begin{array}{c}
\alpha+\beta-1 \\
\alpha-1
\end{array}\right)\right) c_{\alpha+\beta-1, \gamma, a}+\left(\left(\begin{array}{c}
\alpha+\gamma-1 \\
\alpha
\end{array}\right)+\lambda\left(\begin{array}{c}
\alpha+\gamma-1 \\
\alpha-1
\end{array}\right)\right) c_{\beta, \alpha+\gamma-1, a} \\
& -\left(\left(\begin{array}{c}
\beta+\gamma-1 \\
\beta
\end{array}\right)+\lambda\left(\begin{array}{c}
\beta+\gamma-1 \\
\beta-1
\end{array}\right)\right) c_{\alpha, \beta+\gamma-1, a}+\left(\left(\begin{array}{c}
\alpha+a-1 \\
\alpha
\end{array}\right)+\mu\left(\begin{array}{c}
\alpha+a-1 \\
\alpha-1
\end{array}\right)\right) c_{\beta, \gamma, \alpha+a-1} \\
& -\left(\left(\begin{array}{c}
\beta+a-1 \\
\beta
\end{array}\right)+\lambda\left(\begin{array}{c}
\beta+a-1 \\
\beta-1
\end{array}\right)\right) c_{\alpha, \gamma, \beta+a-1}=0
\end{aligned}
$$

where $\alpha+\beta+\gamma+a=k+1, \alpha>\beta \geq 2, \quad \alpha>\gamma$ and $\alpha>a$, obtained from the coefficient of $X^{(\alpha)} Y^{(\beta)(\gamma)(a)}$.

This system can be deduced by a simple computation. Of course, such a system has at least one solution in which the solutions $c_{i, j, l}$ are just the coefficients $\beta_{i, j, l}$ of the coboundaries (23).

The case where $\boldsymbol{v}-\boldsymbol{\mu}-\boldsymbol{\lambda}=3$ : In this case, according to Proposition 4.4 , the space of solutions is spanned by:

$$
c_{4,0,0,}, c_{3,1,0,}, c_{3,0,1,} c_{2,2,0,} c_{2,1,1,} c_{2,0,2}
$$

Moreover, by formula (25), we readily obtain:

$$
-2 c_{2,0,0}+\lambda c_{2,0,0} \lambda c_{2,0,0}+\mu c_{2,0,0}-\mu c_{2,0,0}=0 \text {. }
$$

Thus, we have just proved that the coefficients of every 1-cocycle is expressed in terms of

$$
c_{3,1,0,} c_{3,0,1,}, c_{2,2,0,} c_{2,1,1,} c_{2,0,2}
$$

A direct computation proves that

$$
\begin{aligned}
L_{X} J_{3}^{\lambda, \mu} & =\beta_{2,1,1} X^{\prime \prime} \phi^{\prime} \psi^{\prime}+\beta_{2,2,0}\left(\frac{\lambda}{2} X^{4} \phi \psi+X^{\prime \prime} \phi^{\prime \prime} \psi\right)+\beta_{2,0,2}\left(\frac{\mu}{2} X^{(4)} \phi \psi+X^{\prime \prime} \phi \psi^{\prime \prime}\right) \\
& +\beta_{3,1,0}\left(-\frac{\lambda}{2} X^{(4)} \phi \psi+X^{\prime \prime \prime} \phi^{\prime} \psi\right)+\beta_{3,0,1}\left(-\frac{\mu}{2} X^{(4)} \phi \psi+X^{\prime \prime \prime} \phi \psi^{\prime}\right) .
\end{aligned}
$$

where

$$
\begin{array}{ll}
\beta_{3,1,0}=-(3 \lambda+1) \gamma_{3,0}-\mu \gamma_{1,2} & , \quad \beta_{3,0,1}=-\lambda \gamma_{2,1}-(3 \mu+1) \gamma_{0,3}, \\
\beta_{2,2,0}=-3(\lambda+1) \gamma_{3,0}-\mu \gamma_{2,1} & , \quad \beta_{2,0,2}=-\lambda \gamma_{1,2}-3(\mu+1) \gamma_{0,3}, \\
\beta_{2,1,1}=-(2 \lambda+1) \gamma_{2,1}-(2 \mu+1) \gamma_{1,2} . &
\end{array}
$$

So, for $(\lambda, \mu)=\left(-\frac{1}{3}, 0\right),\left(0,-\frac{1}{3}\right),(-1,0),(0,-1),\left(-\frac{1}{2},-\frac{1}{2}\right)$, the cohomology space is one-dimensional, since only one of the coefficients $c_{3,1,0}$, $c_{3,0,1}, c_{2,2,0}, c_{2,1,1}$ or $c_{2,0,2}$ cannot be eliminated by adding a coboundary. While for $(\lambda, \mu) \neq\left(-\frac{1}{3}, 0\right),\left(0,-\frac{1}{3}\right),(-1,0),(0,-1),\left(-\frac{1}{2},-\frac{1}{2}\right)$, the coeffcient $c_{3,1,0}, c_{3,0,1}, c_{2,2,0}, c_{2,1,1}$ and $c_{2,0,2}$ can be eliminated because $\beta_{3,1,0}, \beta_{3,0,1}, \beta_{2,2,0}$, $\beta_{2,1,1}$ and $\beta_{2,0,2}$ are nonzero. Hence, the cohomology space is zerodimensional.

The case where $\boldsymbol{v}-\boldsymbol{\mu}-\boldsymbol{\lambda}=\mathbf{4}$ : In this case, according to Proposition 4.4, the space of solutions is spanned by:

$$
c_{5,0,0}, c_{4,1,0}, c_{4,0,1}, c_{3,2,0}, c_{3,1,1}, c_{3,0,2}, c_{2,3,0}, c_{2,2,1}, c_{2,1,2}, c_{2,0,3} .
$$

Moreover, by formula (25), we readily obtain:

$$
\begin{aligned}
& -2 c_{4,1,0}+(3 \lambda+1) c_{2,3,0}-(2 \lambda+1) c_{3,2,0}+\mu c_{2,1,2}-\mu c_{3,1,1}=0, \\
& -2 c_{4,0,1}+\lambda c_{2,2,1}-\lambda c_{3,1,1}+(3 \mu+1) c_{2,0,3}-(2 \mu+1) c_{3,0,2}=0, \\
& -5 c_{5,0,0}+\lambda c_{2,3,0}-\lambda c_{4,1,0}+\mu c_{2,0,3}-\mu c_{4,0,1}=0 .
\end{aligned}
$$

Thus, we have just proved that the coefficients of every 1-cocycle is expressed in terms of

$$
c_{3,2,0}, c_{3,1,1}, c_{3,0,2}, c_{2,3,0}, c_{2,2,1}, c_{2,1,2}, c_{2,0,3} \text {. }
$$

A direct computation confirms that, the coefficients of $L_{X} J_{4}^{\lambda, \mu}$ are expressed in terms of:

$$
\begin{array}{lll}
\beta_{2,2,1}=-3(\lambda+1) \gamma_{3,1}-(2 \mu+1) \gamma_{2,2} & , & \beta_{2,1,2}=-(2 \lambda+1) \gamma_{2,2}-3(\mu+1) \gamma_{1,3}, \\
\beta_{3,2,0}=-2(3 \lambda+2) \gamma_{4,0}-\mu \gamma_{2,2} & , & \beta_{3,0,2}=-\lambda \gamma_{2,2}-2(3 \mu+2) \gamma_{0,4}, \\
\beta_{2,3,0}=-2(2 \lambda+3) \gamma_{4,0}-\mu \gamma_{3,1} & , & \beta_{2,0,3}=-\lambda \gamma_{1,3}-2(2 \mu+3) \gamma_{0,4}, \\
\beta_{3,1,1}=-(3 \lambda+1) \gamma_{3,1}-(3 \mu+1) \gamma_{1,3} . &
\end{array}
$$

So, for $\quad(\lambda, \mu)=\left(-\frac{2}{3}, 0\right),\left(0,-\frac{2}{3}\right),\left(-\frac{3}{2}, 0\right),\left(0,-\frac{3}{2}\right),\left(-\frac{1}{2},-1\right),\left(-1,-\frac{1}{2}\right),\left(-\frac{1}{3},-\frac{1}{3}\right)$, the cohomology space is one-dimensional, since only one of the coefficients $c_{3,2,0}, c_{3,1,1}, c_{3,0,2}, c_{2,3,0}, c_{2,2,1}, c_{2,1,2}$ or $c_{2,0,3}$ cannot be eliminated by adding a coboundary. While for $(\lambda, \mu) \neq\left(-\frac{2}{3}, 0\right),\left(0,-\frac{2}{3}\right),\left(-\frac{3}{2}, 0\right), \quad\left(0,-\frac{3}{2}\right),\left(-\frac{1}{2},-1\right),\left(-1,-\frac{1}{2}\right),\left(-\frac{1}{3},-\frac{1}{3}\right), \quad$ the coeffcient $c_{3,2,0}, c_{3,1,1}, c_{3,0,2}, c_{2,3,0}, c_{2,2,1}, c_{2,1,2}$ and $c_{2,0,3}$ can be eliminated because $\beta_{3,2,0}, \beta_{3,1,1}, \beta_{3,0,2}, \beta_{2,3,0}, \beta_{2,2,1}, \beta_{2,1,2}$ and $\beta_{2,0,3}$ are nonzero. Hence, the cohomology space is zero-dimensional.

The case where $\boldsymbol{v}-\boldsymbol{\mu}-\lambda=5$ : In this case, according to Proposition 4.4, the space of solutions is spanned by:

$$
\begin{aligned}
& c_{6,0,0}, c_{5,1,0}, c_{5,0,1}, c_{4,2,0}, c_{4,1,1}, c_{4,0,2}, c_{3,3,0}, c_{3,2,1}, \\
& c_{3,1,2}, c_{3,0,3}, c_{2,4,0}, c_{2,3,1}, c_{2,2,2}, c_{2,1,3}, c_{2,0,4} .
\end{aligned}
$$

Moreover, by formula (25), we readily obtain:

$$
\begin{aligned}
& -2 c_{4,1,1}+(3 \lambda+1) c_{2,3,1}-(2 \lambda+1) c_{3,2,1}+(3 \mu+1) c_{2,1,3}-(2 \mu+1) c_{3,1,2}=0, \\
& -5 c_{5,1,0}+(4 \lambda+1) c_{2,4,0}-(2 \lambda+1) c_{4,2,0}+\mu c_{2,1,3}-\mu c_{4,1,1}=0, \\
& -5 c_{5,0,1}+\lambda c_{2,3,1}-\lambda c_{4,1,1}+(4 \mu+1) c_{2,0,4}-(2 \mu+1) c_{4,0,2}=0, \\
& -2 c_{4,2,0}+2(3 \lambda+2) c_{2,4,0}-3(\lambda+1) c_{3,3,0}+\mu c_{2,2,2}-\mu c_{3,2,1}=0, \\
& -2 c_{4,0,2}+\lambda c_{2,2,2}-\lambda c_{3,1,2}+2(3 \mu+2) c_{2,0,4}-3(\mu+1) c_{3,0,3}=0, \\
& -9 c_{6,0,0}+\lambda c_{2,4,0}-\lambda c_{5,1,0}+\mu c_{2,0,4}-\mu c_{5,0,1}=0, \\
& -5 c_{6,0,0}+\lambda c_{3,3,0}-\lambda c_{4,2,0}+\mu c_{3,0,3}-\mu c_{4,0,2}=0 .
\end{aligned}
$$


Thus, we have just proved that the coefficients of every 1-cocycle is expressed in terms of

$$
c_{2,4,0}, c_{2,0,4}, c_{3,3,0}, c_{3,0,3}, c_{3,2,1}, c_{3,1,2}, c_{2,3,1}, c_{2,2,2} \text {. }
$$

A direct computation confirms that, the coefficients of $L_{X} J_{5}^{\lambda, \mu}$ are expressed in terms of:

$$
\begin{array}{lll}
\beta_{3,2,1}=-2(3 \lambda+2) \gamma_{4,1}-(3 \mu+1) \gamma_{2,3} & , & \beta_{3,1,2}=-(3 \lambda+1) \gamma_{3,2}-2(3 \mu+2) \gamma_{1,4}, \\
\beta_{2,3,1}=-2(2 \lambda+3) \gamma_{4,1}-(2 \mu+1) \gamma_{3,2} & , & \beta_{2,2,2}=-3(\lambda+1) \gamma_{3,2}-3(\mu+1) \gamma_{2,3}, \\
\beta_{3,3,0}=-10(\lambda+1) \gamma_{5,0}-\mu \gamma_{3,2} & , & \beta_{3,0,3}=-\lambda \gamma_{2,3}-10(\mu+1) \gamma_{0,5}, \\
\beta_{2,4,0}=-5(\lambda+2) \gamma_{5,0}-\mu \gamma_{4,1} & , & \beta_{2,0,4}=-\lambda \gamma_{1,4}-5(\mu+2) \gamma_{0,5} .
\end{array}
$$

So, for $\quad(\lambda, \mu)=\left(-\frac{2}{3},-\frac{1}{3}\right),\left(-\frac{1}{3},-\frac{2}{3}\right),\left(-\frac{3}{2},-\frac{1}{2}\right),(-1,-1),(-1,0),(0,-1),(-2,0),(0,-2)$, the cohomology space is one-dimensional, since only one of the coefficients $\quad c_{2,4,0}, c_{2,0,4}, c_{3,3,0}, c_{3,0,3}, c_{3,2,1}, c_{3,1,2}, c_{2,3,1}$ or $c_{2,2,2}$ cannot be eliminated by adding a coboundary. While for $(\lambda, \mu) \neq\left(-\frac{2}{3},-\frac{1}{3}\right),\left(-\frac{1}{3},-\frac{2}{3}\right),\left(-\frac{3}{2},-\frac{1}{2}\right),(-1,-1),(-1,0),(0,-1),(-2,0),(0,-2)$, the coeffcient $c_{2,4,0}, c_{2,0,4}, c_{3,3,0}, c_{3,0,3}, c_{3,2,1}, c_{3,1,2}, c_{2,3,1}$ and $c_{2,2,2}$ can be eliminatedbecause $\beta_{2,4,0}, \beta_{2,0,4}, \beta_{3,3,0}, \beta_{3,0,3}, \beta_{3,2,1}, \beta_{3,1,2}, \beta_{2,3,1}$ and $\beta_{2,2,2}$ are nonzero. Hence, the cohomology space is zero-dimensional.

The case where $\boldsymbol{\nu}-\boldsymbol{\mu}-\boldsymbol{\lambda}=\mathbf{6}$ : In this case, according to Proposition 4.4 together with formulas (25), we check that the coefficients of every 1 -cocycle are expressed in terms of

$$
c_{4,2,1}, c_{4,0,3}, c_{3,2,2}, c_{3,1,3}, c_{3,0,4}, c_{2,5,0}, c_{2,4,1}, c_{2,3,2}, c_{2,1,4} \text {. }
$$

A direct computation confirms that, the coefficients of $L_{X} J_{6}^{\lambda, \mu}$ are expressed in terms of:

$$
\beta_{4,2,1}, \beta_{4,0,3}, \beta_{3,2,2}, \beta_{3,1,3}, \beta_{3,0,4}, \beta_{2,5,0}, \beta_{2,4,1}, \beta_{2,3,2}, \beta_{2,1,4} \text {. }
$$

So, in the same way as before, by Lemma 5.2 , we can see, with the help of the maple, that the cohomology space is given as in (17).

The case where $\boldsymbol{\nu}-\boldsymbol{\mu}-\lambda=7$ : In this case, according to Proposition 4.4 together with formulas (25), we check that the coefficients of every 1 -cocycle are expressed in terms of

$$
c_{8,0,0}, c_{7,1,0}, c_{7,0,1}, c_{5,3,0}, c_{5,0,3}, c_{4,0,4}, c_{2,4,2}, c_{2,1,5}, c_{2,0,6} \text {. }
$$

A direct computation confirms that, the coefficients of $L_{X} J_{7}^{\lambda, \mu}$ are expressed in terms of:

$$
\beta_{8,0,0}, \beta_{7,1,0}, \beta_{7,0,1}, \beta_{5,3,0}, \beta_{5,0,3}, \beta_{4,0,4}, \beta_{2,4,2}, \beta_{2,1,5}, \beta_{2,0,6} \text {. }
$$

So, in the same way as before, by Lemma 5.2 , we can see, with the help of the maple, that the cohomology space is given as in (18).

The case where $\boldsymbol{v}-\boldsymbol{\mu}-\boldsymbol{\lambda}=\mathbf{8}$ : In this case, according to Proposition 4.4 together with formulas (25), we check that the coefficients of every 1-cocycle are expressed in terms of:

$$
c_{9,0,0}, c_{8,0,1}, c_{7,2,0}, c_{7,0,2}, c_{6,3,0}, c_{6,0,3}, c_{5,3,1}, c_{3,0,6}, c_{2,0,7} .
$$

A direct computation confirms that, the coefficients of $L_{X} J_{8}^{\lambda, \mu}$ are expressed in terms of:

$$
\beta_{9,0,0}, \beta_{8,0,1}, \beta_{7,2,0}, \beta_{7,0,2}, \beta_{6,3,0}, \beta_{6,0,3}, \beta_{5,3,1}, \beta_{3,0,6}, \beta_{2,0,7} .
$$

So, in the same way as before, by Lemma 5.2 , we can see, with the help of the maple, that the cohomology space is given as in (19).

The case where $\boldsymbol{v}-\boldsymbol{\mu}-\boldsymbol{\lambda}=\mathbf{9}$ : In this case, according to Proposition 4.4 together with formulas (25), we check that the coefficients of every 1 -cocycle are expressed in terms of

$$
c_{10,0,0}, c_{9,0,1}, c_{8,2,0}, c_{6,4,0}, c_{6,0,4}, c_{5,3,2}, c_{3,0,7}, c_{2,0,8} \text {. }
$$

A direct computation confirms that, the coefficients of $L_{X} J_{9}^{\lambda, \mu}$ are expressed in terms of:

$$
\beta_{10,0,0}, \beta_{9,0,1}, \beta_{8,2,0}, \beta_{6,4,0}, \beta_{6,0,4}, \beta_{5,3,2}, \beta_{3,0,7}, \beta_{2,0,8} .
$$

So, in the same way as before, by Lemma 5.2 , we can see, with the help of the maple, that the cohomology space is given as in (20).

The case where $\boldsymbol{v}-\boldsymbol{\mu}-\boldsymbol{\lambda}=\mathbf{9}$ : In this case, according to Proposition 4.4 together with formulas (25), we check that the coefficients of every 1 -cocycle are expressed in terms of

$$
c_{11,0,0}, c_{10,0,1}, c_{9,2,0}, c_{6,5,0}, c_{6,0,5}, c_{5,3,3}, c_{3,0,8}, c_{2,0,9} \text {. }
$$

A direct computation confirms that, the coefficients of $L_{X} J_{10}^{\lambda, \mu}$ are expressed in terms of:

$$
\beta_{11,0,0}, \beta_{10,0,1}, \beta_{9,2,0}, \beta_{6,5,0}, \beta_{6,0,5}, \beta_{5,3,3}, \beta_{3,0,8}, \beta_{2,0,9} .
$$

So, in the same way as before, by Lemma 5.2 , we can see, with the help of the maple, that the cohomology space is given as in (21).

The case where $\boldsymbol{\nu}-\boldsymbol{\mu}-\boldsymbol{\lambda}=\mathbf{1 1}$ : In this case, according to Proposition 4.4 together with formulas (25), we check that the coefficients of every 1 -cocycle are expressed in terms of

$$
c_{12,0,0}, c_{11,0,1}, c_{10,0,2}, c_{9,0,3}, c_{7,0,5}, c_{5,7,0}, c_{5,0,7}, c_{2,1,9} \text {. }
$$

A direct computation confirms that, the coefficients of $L_{X} J_{11}^{\lambda, \mu}$ are expressed in terms of:

$$
\beta_{12,0,0}, \beta_{11,0,1}, \beta_{10,0,2}, \beta_{9,0,3}, \beta_{7,0,5}, \beta_{5,7,0}, \beta_{5,0,7}, \beta_{2,1,9} .
$$

So, in the same way as before, by Lemma 5.2, we can see, with the help of the maple, that the cohomology space is given as in (22). This completes the proof.

\section{Conjecture 5.1}

For $v-\mu-\lambda \in \mathbb{N}+12, \lambda$ and $\mu$ are generic, one hase

$$
\mathrm{H}_{\text {diff }}^{1}\left(\operatorname{Vect}\left(\mathbb{R P}^{1}\right), \mathfrak{a f f}(1) ; \mathcal{D}_{\lambda, \mu ; \nu}\right)=0 \text {. }
$$

\section{Conclusion}

In this paper, we classify afff(1) -invariant linear differential operators from $\operatorname{Vect}\left(\mathbb{R}^{1}\right)$ to $\mathcal{D}_{\mu ; \nu}$ vanishing on aff(1), where $\mathcal{D}_{\mu, \nu}:=\operatorname{Hom} \operatorname{diff}\left(\mathcal{F}_{\lambda} \otimes \mathcal{F} ; \mathcal{F}_{v}\right)$ is the space of bilinear differential operators acting on weighted densities. This result allows us to compute the first differential $\mathfrak{a} \mathfrak{f} \mathfrak{f}(1)$-relative cohomology of $\operatorname{Vect}\left(\mathbb{R P}^{1}\right)$ with coefficients in $\mathcal{D}_{\lambda, \mu ; j}$.

\section{References}

1. Fuks DB (1986) Cohomology of infinite-dimensional Lie algebras. Contemp Soviet Math, Consultants Bureau, New-York.

2. Feigin BL, Fuks DB (1982) Homology of Lie algebras on vector fields on the line. Funkts Anal Prilozhen 16: 47-63.

3. Bouarroudj S, Ovsienko V (1998) Three cocycle on Diff( $\left.\mathrm{S}^{1}\right)$ generalizing the Schwarzian derivative. Int Math Res Notices 25-39.

4. Wilczynski EJ (1906) Projective differential geometry of curves and ruled surfaces, Leipzig-Teubner.

5. Bouarroudj S (2007) On sl(2)-relative Cohomology of the Lie algebra of vector fields and differentiel operators. J Nonlinear Math Phys 14: 1-29.

6. Bouarroudj S (2006) Projective and conformal Schwarzian derivatives and cohomology of Lie algebras vector fields related to differential operators. In Jour Geom Methods Mod Phys 3: 667-696. 
Citation: Meher A (2017) On the First aff(1)-Relative Cohomology of the Lie Algebra of Vector Fields and Differential Operators. J Generalized Lie Theory Appl 11: 269. doi: 10.4172/1736-4337.1000269

Page 6 of 6

7. Lecomte PBA, Ovsienko V (2000) Cohomology of the vector fields Lie algebra and modules of differential operators on a smooth manifold. Compositio Mathematica 124: 95-110.
8. Omri S (2016) On aff(1)-relative cohomology of the Lie algebra of vector fields on weighted densities on R", Math. Reports 18: 509-514. 